\title{
Dexmedetomidine pretreatment attenuates propofol-induced neurotoxicity in neuronal cultures from the rat hippocampus
}

\author{
YI WEI $^{1}$, JUNHUA HU ${ }^{2}$, YUBING LIANG ${ }^{3}$, YULING ZHONG ${ }^{1}$, DAN HE $^{1}$, \\ YI QIN ${ }^{1}$, LI LI $^{1}$, JING CHEN $^{1}$, QIANG XIAO ${ }^{4}$ and YUBO XIE ${ }^{1}$ \\ ${ }^{1}$ Department of Anesthesiology, The First Affiliated Hospital of Guangxi Medical University, Nanning, Guangxi 530021; \\ ${ }^{2}$ Department of Anesthesiology, The Affiliated Hospital of Guilin Medical University, Guilin, Guangxi 541004; \\ ${ }^{3}$ Department of Anesthesiology, The Affiliated Tumor Hospital of Guangxi Medical University, Guangxi 524001; \\ ${ }^{4}$ Department of Surgery, The First Affiliated Hospital of Guangxi Medical University, Guangxi 530021, P.R. China
}

Received August 3, 2015; Accepted July 4, 2016

DOI: $10.3892 / \mathrm{mmr} .2016 .5628$

\begin{abstract}
Propofol is widely used for the induction and maintenance of pediatric anesthesia. Previous studies have indicated that propofol can induce apoptosis, and damage cognitive and memory functions. Dexmedetomidine is a potent $\alpha-2$ adrenoceptor agonist with high selectivity. Previous observations have shown that dexmedetomidine exhibits anti-apoptotic qualities. The present study evaluated the neuroprotective effects of dexmedetomidine pretreatment against propofol-induced neurotoxicity in immature hippocampal neurons. The viability and apoptotic rate of the neurons were detected using a Cell Counting Kit- 8 assay and flow cytometry. The mRNA and protein expression levels of brain-derived neurotrophic factor (BDNF), B-cell lymphoma-2 (Bcl-2) and phosphorylated-cyclic-AMP response element binding protein (p-CREB) were detected using semiquantitative reverse transcription-polymerase chain reaction and western blot analyses, respectively. These results showed that propofol exposure $(100 \mu \mathrm{M} ; 3 \mathrm{~h})$ reduced neuronal viability, induced cell apoptosis and decreased the expression levels of BDNF, Bcl-2 and p-CREB. Dexmedetomidine treatment $(0.001-100 \mu \mathrm{M})$ of the neurons prior to propofol exposure attenuated the propofol-induced neuronal apoptosis and increased expression levels of BDNF, Bcl-2 and p-CREB compared with the propofol only group. In addition, dexmedetomidine at the highest concentration provided superior neuroprotection of neurons. These in vitro data indicated that dexmedetomidine exerted direct neuroprotective effects to prevent cultured hippocampal neuronal injury caused by
\end{abstract}

Correspondence to: Professor Yubo Xie, Department of Anesthesiology, The First Affiliated Hospital of Guangxi Medical University, 6 Shuang-Yong Road, Nanning, Guangxi 530021, P.R. China

E-mail: xieyubo715001@aliyun.com

Key words: hippocampal neurons, apoptosis, dexmedetomidine, propofol, neuroprotection propofol, accompanied by an increase in the levels of $\mathrm{p}-\mathrm{CREB}$, Bcl-2 and BDNF.

\section{Introduction}

There has been an increase in the number of studies examining the possible detrimental effects of anesthesia in the developing brain. Propofol (2,6-diisopropylphenol) is a sedative-hypnotic agent widely used for the induction and maintenance of anesthesia in surgery, and sedation in intensive care units. It has been reported that propofol exposure can induce cell death in neural precursor or stem cells (1), immature hippocampal neurons (2) and cortical cells (3) in vitro. Several in vivo studies have also demonstrated that propofol can cause neuronal cell apoptosis in the developing brain of rodents $(4,5)$ and non-human primates (NHPs) (6). Therefore, it is important to develop promising strategies for protection of the developing brain from the potentially deleterious effects of propofol.

Dexmedetomidine is an $\alpha-2$ adrenoceptor agonist with high selectivity, and with sympatholytic, sedative and analgesic properties. It is considered to offer potential benefits towards neuroprotection (7). In a murine model of perinatal excitotoxic brain damage, dexmedetomidine has been found to provide potent neuroprotection (8). Previous in vitro and in vivo observations have demonstrated that dexmedetomidine protects against neuroapoptosis induced by isoflurane in the hippocampus of neonatal rats $(9,10)$. Isoflurane is a type of volatile anesthetic and it is reported to cause a similar pattern of neuronal apoptosis as propofol in the neonatal brain (6). Anesthesia-induced apoptotic damage in the developing brain is regulated, at least in part, by the brain-derived neurotrophic factor (BDNF)-modulated apoptotic cascade (11). In addition, it has been reported that the neuroprotective effects of dexmedetomidine are mediated by upregulating the levels of BDNF, phosphorylated-cyclic-AMP response element binding protein (p-CREB) $(12,13)$ and the antiapoptotic factor, B-cell lymphoma 2 (Bcl-2) (14).

The present study used neuronal cultures from the rat hippocampus to investigate whether dexmedetomidine pretreatment is able to effectively attenuate propofol-induced 
neurotoxicity in vitro. The study also aimed to examine alterations in the expression levels of p-CREB, Bcl-2 and BDNF following exposure to propofol and dexmedetomidine.

\section{Materials and methods}

Hippocampal neuronal culture and drug treatment. The experimental procedure was approved by the Animal Use and Care Committee of Guangxi Medical University (Guangxi, China) and performed in strict accordance with the guidelines of the National Institutes of Health Guide for the Use of Laboratory Animals. Primary hippocampal cultures were prepared, as described previously (15). In brief, 40 female Sprague-Dawley rats (age, 4 months; weight, 400-450 g) in advanced pregnancy (Guangxi Medical University Laboratory Animal Co.; permission no. SCXK 2009-0002) were housed under standard conditions with a 12-h light/dark cycle at $23 \pm 2^{\circ} \mathrm{C}, 50 \pm 5 \%$ relative humidity and free access to food and water. The rats were then anesthetized using $10 \%$ (w/v) chloral hydrate $(3.5 \mathrm{ml} / \mathrm{kg}$; Sigma-Aldrich, Merck Millipore, Darmstadt, Germany) and embryonic day 16-18 fetuses were removed. Next, the pregnant rats were sacrificed by cervical dislocation. The fetuses were sacrificed by rapid decapitation, followed by immediate removal of the brain and its surrounding membranes. The hippocampus was then rapidly dissected from the cortex. The meningeal tissues were removed and the hippocampus was dissociated mechanically into $1 \mathrm{~mm}^{3}$ pieces. Equal volume of $0.25 \%$ trypsin solution (Sigma-Aldrich, Merck Millipore) was added to the dissected tissue and incubated at $37^{\circ} \mathrm{C}$ for $15 \mathrm{~min}$, mixing every $5 \mathrm{~min}$. Following the removal of the trypsin solution, $1 \mathrm{ml}$ precooled fetal calf serum (FCS; Gibco; Thermo Fisher Scientific, Inc., Waltham, MA, USA) was added into the tissue, mixed gently, and incubated in a $37^{\circ} \mathrm{C}$ water bath for $5 \mathrm{~min}$ to stop the digestion. The tissues were centrifuged for $5 \mathrm{~min}$ at $106 \mathrm{x} \mathrm{g}$ and the supernatant was discarded. Cells were washed three times and resuspended in plating medium [Neurobasal medium (Gibco; Thermo Fisher Scientific, Inc.) supplemented with $10 \%$ FCS, $0.2 \mathrm{mM}$ glutamine, $100 \mathrm{U} / \mathrm{ml}$ penicillin and $100 \mathrm{U} / \mathrm{ml}$ streptomycin] and were transferred as a $0.5 \mathrm{ml}$ aliquot to a tube that contained $0.5 \mathrm{ml}$ of $4 \%$ Typan blue. The number of Trypan blue-excluding cells were counted by inverted microscope (Olympus Corporation, Tokyo, Japan). The number of Trypan blue-excluding cells were counted, and the cells were plated onto six-well culture plates (Corning, Inc., Acton, MA, USA) previously coated with poly-L-lysine $(0.1 \mathrm{mg} / \mathrm{ml}$, Gibco; Thermo Fisher Scientific, Inc.) at a density of $5 \times 10^{4}$ cells $/ \mathrm{ml}$. The cultures were maintained at $37^{\circ} \mathrm{C}$ with $5 \% \mathrm{CO}_{2}$, supplemented with Neurobal medium with 2\% B-27 (Gibco; Thermo Fisher Scientific, Inc.), $2 \mathrm{mmol} / \mathrm{ml}$ glutamine, penicillin $(100 \mathrm{U} / \mathrm{ml})$ and streptomycin $(100 \mu \mathrm{g} / \mathrm{ml})$. Half the cell culture media was replaced every 3 days. Immunocytochemical staining was performed on neurons maintained for 8 days in vitro (DIV 8) using mouse monoclonal antibody against NeuN (cat. no. ABN78; Chemicon, Temecula, CA, USA). The cells adhered to and grew on the coverslips. Following fixing with $4 \%$ paraformaldehyde, coverslips bearing the neuronal cultures were pre-incubated in 5\% goat serum in phosphate-buffered saline (PBS) supplemented with $0.2 \%$ Triton-X 100 for $1 \mathrm{~h}$ at room temperature, followed by incubation with the primary antibody (1:500 dilution) overnight at $4^{\circ} \mathrm{C}$. Binding of the NeuN antibody was detected with a goat anti-mouse IgG biotinylated secondary antibody (1:5,000; cat. no. ab32096; Abcam, Cambridge, MA, USA). 3,3'-Diaminobenzidine tetrahydrochloride was used as the substrate. Staining for NeuN was then visualized using an AxioM1 light microscope (BX53; Olympus Corporation).

The DIV 8 primary hippocampal cultures were used for the drug exposure experiments in the present study. The cells were seeded at a density of $5.0 \times 10^{4}$ cells/well and were assigned to a control group, propofol group and dexmedetomidine + propofol groups. The cells in the control group were incubated without drugs in an intralipid vehicle (Baxter, Guangzhou, China) at $37^{\circ} \mathrm{C}$ for $3 \mathrm{~h}$, whereas the cells in the propofol group were incubated with $100 \mu \mathrm{M}$ propofol for $3 \mathrm{~h}$ at $37^{\circ} \mathrm{C}$ in the absence of dexmedetomidine pretreatment. The cells in the dexmedetomidine + propofol groups were incubated with $0.001,0.01,0.1,1,10$ or $100 \mu \mathrm{M}$ dexmedetomidine, respectively, at $37^{\circ} \mathrm{C}$ for $30 \mathrm{~min}$, following which $100 \mu \mathrm{M}$ propofol was added to the culture medium at $37^{\circ} \mathrm{C}$ for $3 \mathrm{~h}$. The viability and apoptotic rate of the neurons were then detected using a Cell Counting Kit-8 (CCK-8) assay and flow cytometry. The expression levels of BDNF, Bcl-2 and p-CREB were detected using semiquantitative reverse transcription-polymerase chain reaction (RT-PCR) and western blot analyses.

Cell viability assay. Cell viability was assessed using a CCK-8 assay (Dojindo Molecular Technologies, Inc., Kumamoto, Japan). The cells were incubated with $100 \mu \mathrm{M}$ propofol for $3 \mathrm{~h}$ in the absence or presence of dexmedetomidine pretreatment, following which CCK-8 solution was added and the culture was incubated for $2 \mathrm{~h}$ under $5 \% \mathrm{CO}_{2}$ at $37^{\circ} \mathrm{C}$. The absorbance was read at $450 \mathrm{~nm}$ on a microplate reader (Thermo Fisher Scientific, Inc.), with the value directly proportional to the number of viable cells in the culture medium.

Flow cytometric analysis. Flow cytometric analysis was performed using an Annexin V-fluorescein isothiocyanate (FITC) apoptosis detection kit (KeyGen, Nanjing, China) according to the manufacturer's protocol. Primary hippocampal neurons were briefly trypsinized and then washed twice with cold $1 \mathrm{X}$ PBS. The cells were pelleted by centrifugation at $425 \mathrm{x}$ g for $5 \mathrm{~min}$ at $4^{\circ} \mathrm{C}$ and resuspended in $1 \mathrm{X}$ binding buffer followed by incubation with staining solution of annexin V-FITC and propidium iodide (PI) for $10 \mathrm{~min}$ in the dark at $4{ }^{\circ} \mathrm{C}$. The samples were maintained on ice during the entire procedure and analyzed immediately using flow cytometry. The cells from each sample (10,000 cells) were scanned and analyzed using a FACS Calibur flow cytometer (Becton Dickinson; BD Biosciences, San Diego, CA, USA). Necrosis and apoptosis were determined by PI (FL2) and annexin V-FITC (FL1) fluorescence, respectively. The percentages of apoptotic cells in each sample were estimated.

Semiquantitative RT-PCR. Total RNA from primary hippocampal neurons in each of the different groups was extracted using TRIzol reagent (Invitrogen; Thermo Fisher Scientific, Inc.), according to the manufacturer's protocol. The reagents 
for semiquantitative RT-PCR were those supplied with the RevertAid $^{\mathrm{TM}}$ First Strand cDNA Synthesis kit (Fermentas; Thermo Fisher Scientific, Inc.). First-strand cDNA was generated from the total RNA $(2 \mu \mathrm{g})$ by reverse transcription. PCR was performed in a total volume of $25 \mu \mathrm{l}(2 \mu \mathrm{l}$ cDNA, $0.5 \mu \mathrm{l}$ of $10 \mu \mathrm{M}$ each primer, $10 \mu \mathrm{l}$ PCR master mix and $12 \mu \mathrm{l}$ sterilized, deionized water). The PCR reaction conditions were as follows: Denaturation at $94^{\circ} \mathrm{C}$ for $45 \mathrm{sec}$, annealing at $59.1^{\circ} \mathrm{C}$ (BDNF), $55.6^{\circ} \mathrm{C}(\mathrm{Bcl}-2)$ or $57.5^{\circ} \mathrm{C}(\mathrm{GAPDH})$ for $30 \mathrm{sec}$, and extension at $72^{\circ} \mathrm{C}$ for $30 \mathrm{sec}$. Primer sequences were as follows: BDNF, forward 5'-AGCCTCCTCTGCTCTTTC TGCTGGA-3' and reverse 5'-CTTTTGTCTATGCCCCTG CAGCCTT-3'; Bcl-2, forward 5'-GGTGGTGGAGGAACT CTTCA-3' and reverse 5'-CTCACTTGTGGCCCAGGTAT-3'; GAPDH, forward 5'-ACAGCAACAGGGTGGTGGAC-3' and reverse 5'-TTTGAGGGTGCAGCGAACTT-3'. Following PCR amplification, the products were electrophoresed and separated on a $1.5 \%$ agarose gel stained with ethidium bromide. Densitometric analyses of bands for specific genes were performed and normalized to the level of the endogenous control mRNA (GAPDH) using Quantity One v5.0 software (Bio-Rad, Berkeley, CA, USA).

Western blot analysis. Following incubation, the cell proteins were extracted on ice for $60 \mathrm{~min}$ in Western and IP lysis buffer (Beyotime Institute of Biotechnology, Shanghai, China), which was added to PMSF (1 mM) prior to use and supplemented with protease inhibitor cocktail. Following centrifugation at $4^{\circ} \mathrm{C}$ at $17,254 \mathrm{x}$ g for $10 \mathrm{~min}$, the total lysates were separated on $10 \%$ SDS-PAGE gels (30 mg/lane; Solarbio, Beijing, China), and electrophoretically transferred onto polyvinylidene fluoride membranes $(0.22 \mu \mathrm{m}$; EMD Millipore, Billerica, MA, USA). The membranes were blocked in Tris-buffered saline/0.1\% Tween-20/5\% non-fat milk for $1 \mathrm{~h}$ at room temperature, and were then incubated with primary antibodies against Bcl-2 (1:1,000; cat. no. ab32096; Abcam), BDNF (1:1,000; cat. no. ab108319; Abcam), pCREB (1:1,000; cat. no. ab32096; Abcam) and GAPDH (1:5,000; cat. no. sc-25778; Santa Cruz Biotechnology, Inc.) overnight at $4^{\circ} \mathrm{C}$. The membranes were then rinsed in PBS with $0.1 \%$ Tween 20 and incubated with horseradish peroxidase-conjugated secondary antibody (1:5,000; cat. no. sc-25778; Santa Cruz Biotechnology, Inc. Dallas, TX, USA) for $30 \mathrm{~min}$ at room temperature. Images of the immunoblots were analyzed using Quantity One V5.0 software (Bio-Rad). The level of each protein was normalized with respect to GAPDH, the domestic loading control.

Statistical analysis. SPSS 19 (IBM SPSS, Armonk, NY, USA) and Origin 7.5 (OriginLab, Northampton, MA, USA) were used for statistical analysis. The data are expressed as the mean \pm standard deviation, and one-way analysis of variance was performed to estimate significant differences among groups. $\mathrm{P}<0.05$ was considered to indicate a statistically significant difference.

\section{Results}

Hippocampal neuron culture. In the present study, immunocytochemical staining with NeuN was used to identify putative

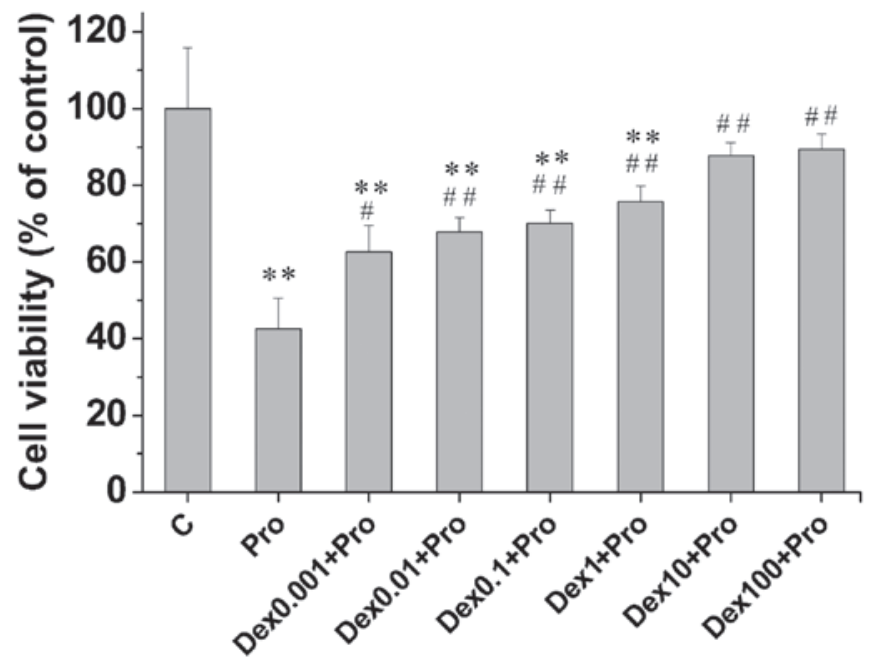

Figure 1. Dex attenuates Pro-induced reductions in neuronal viability in neuronal cultures from the rat hippocampus. Cells were exposed to Pro $(100 \mu \mathrm{M} ; 3 \mathrm{~h})$ in the absence or presence of Dex pretreatment $(0.001,0.01$, $0.1,1,10$ and $100 \mu \mathrm{M})$. The viability of the cells was detected using a Cell Counting Kit- 8 assay. Data are presented as the mean \pm standard deviation of values obtained from four culture wells per experiment, determined in three independent experiments. ${ }^{* *} \mathrm{P}<0.01$, compared with group $\mathrm{C},{ }^{*} \mathrm{P}<0.05$ and ${ }^{\# \#} \mathrm{P}<0.01$, compared with group Pro. Dex, dexmedetomidine; C, control; Pro, propofol.

neurons, which revealed a population of neuronal cells with $>90 \%$ purity. These results showed that the hippocampal neurons had been cultured successfully.

Propofol reduces neuronal viability and induces apoptosis in neuronal cultures from the rat hippocampus. The DIV 8 primary hippocampal neurons in the propofol group were exposed to propofol (100 $\mu \mathrm{M}$ for $3 \mathrm{~h}$ ) in the absence of dexmedetomidine pretreatment. Neuronal injury was then determined using a CCK-8 assay and flow cytometric analysis. The results of the CCK-8 assay revealed that the neuronal viability in the propofol-treated group was reduced by $57.4 \%$, compared with that in the control group (Fig. 1). An increase in the percentage of apoptotic cells was also observed in the propofol-treated cells (31.05 $\pm 4.33 \%)$, compared with the control cells $(12.26 \pm 2.48 \%)$, as shown in Fig. 2A and B.

Dexmedetomidine attenuates propofol-induced apoptosis and increases neuronal viability in neuronal cultures from the rat hippocampus. The DIV 8 primary hippocampal neurons in the propofol + dexmedetomidine groups were pretreated with different concentrations $(0.001,0.01,0.1,1,10$ and $100 \mu \mathrm{M})$ of dexmedetomidine, prior to propofol exposure (100 $\mu \mathrm{M}$ for $3 \mathrm{~h}$ ), and subjected to a CCK-8 assay and flow cytometric analysis. Dexmedetomidine significantly increased neuronal viability, by up $110 \%$, compared with propofol exposure without dexmedetomidine pretreatment $(\mathrm{P}<0.05$; Fig. 1). No significant differences in cell viability were found among the $10-100 \mu \mathrm{M}$ dexmedetomidine-pretreated cells and the control cells ( $\mathrm{P}>0.05$; Fig. 1$)$. In the cells pretreated with $0.001,0.01,0.1,1,10$ and $100 \mu \mathrm{M}$ dexmedetomidine, the apoptotic rates of the cells were 23.16 \pm 3.06 , $22.83 \pm 3.17,20.67 \pm 3.35,18.75 \pm 2.76,17.53 \pm 2.92$ and $15.47 \pm$ $2.59 \%$, respectively. Pretreatment with dexmedetomidine at 
A

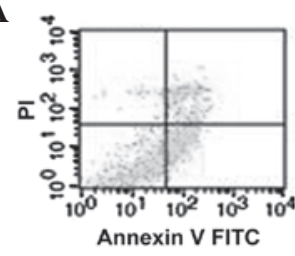

$\mathrm{C}$

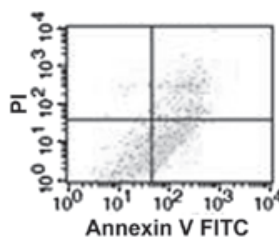

Dex0.1+Pro

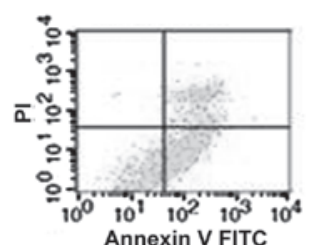

Pro

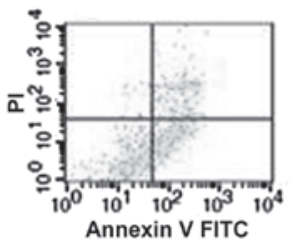

Dex1+Pro

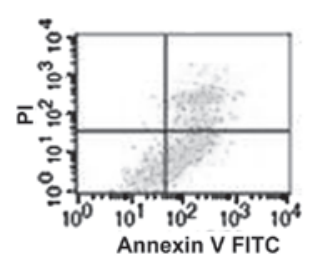

Dex0.001+Pro

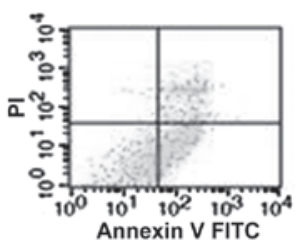

Dex $10+$ Pro

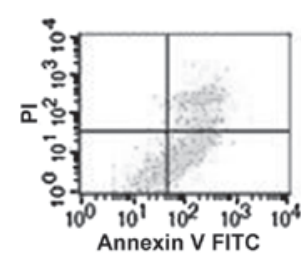

Dex $0.01+$ Pro

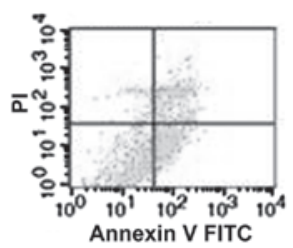

Dex100+Pro

B

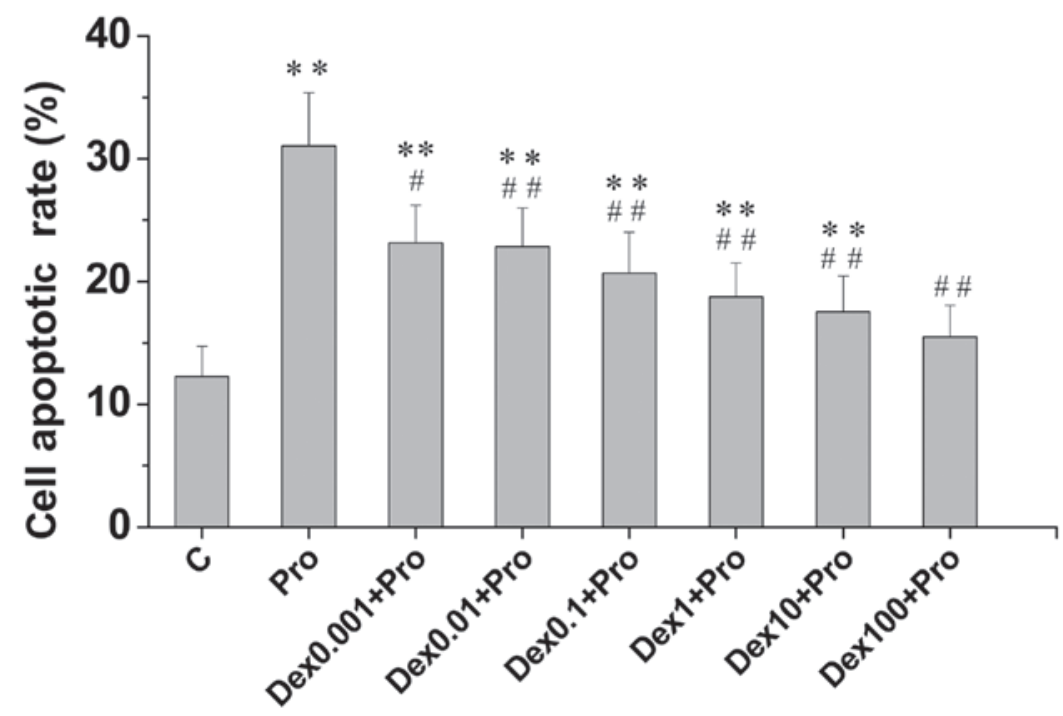

Figure 2. Dex attenuates Pro-induced apoptosis in immature hippocampal neurons. The cells ( 8 days in vitro) were exposed to Pro (100 $\mu \mathrm{M}, 3 \mathrm{~h})$ in the absence or presence of Dex pretreatment $(0.001,0.01,0.1,1,10$ and $100 \mu \mathrm{M})$. Apoptotic cells were analyzed using flow cytometry. (A) Hippocampal neurons were stained with annexin V-FITC and PI. (B) Percentages of apoptotic neurons in the control cultures and each separate experimental culture were determined. Data are presented as the means \pm standard deviation of values from triplicate independent experiments."* $\mathrm{P}<0.01$, compared with group $\mathrm{C}$; ${ }^{\#} \mathrm{P}<0.05$ and ${ }^{\#} \mathrm{P}<0.01$, compared with group Pro. Dex, dexmedetomidine; C, control; Pro, propofol; FITC, fluorescein isothiocyanate; PI. propidium iodide.

all concentration levels assessed in the present study resulted in fewer apoptotic cells, compared with propofol exposure in the absence of dexmedetomidine pretreatment $(31.05 \pm 4.33 \%$; $\mathrm{P}<0.05)$. No significant difference was observed in apoptotic rates between the $100 \mu \mathrm{M}$ dexmedetomidine-pretreated cells and the control cells $(\mathrm{P}>0.05$; Fig. 2B).

Propofol decreases the levels of BDNF, Bcl-2 and p-CREB in hippocampal neurons. The present study evaluated the effects of propofol exposure on the mRNA expression levels of BDNF and $\mathrm{Bcl}-2$, known to be important in cell survival, using a semiquantitative RT-PCR method. Compared with the control cells, the mRNA levels of Bcl-2 (Fig. 3A and B) and BDNF (Fig. 3C and D) in the cells treated with propfol were reduced by 72.4 and $60.3 \%$, respectively $(\mathrm{P}<0.05)$. Consistent with the results of the semiquantitative RT-PCR analysis, western blot analysis (Fig. 4A) revealed that the protein levels of BDNF (Fig. 4B) and Bcl-2 (Fig. 4C) in the cells treated with propofol were reduced by 63.5 and $64.5 \%$, respectively, compared with control cells $(\mathrm{P}<0.05)$. Propofol also significantly decreased the protein level of p-CREB, by $59.0 \%$, compared with the control ( $\mathrm{P}<0.05$; Fig. 4D).

Dexmedetomidine attenuates the propofol-induced reduction in the expression levels of BDNF, Bcl-2 and p-CREB in hippocampal neurons. The semiquantitative RT-PCR analysis revealed that the treatment of DIV 8 primary hippocampal neurons with dexmedetomidine at concentrations of $0.001,0.01,0.1,1,10$ and $100 \mu \mathrm{M}$, prior to propofol exposure $(100 \mu \mathrm{M}$ for $3 \mathrm{~h})$, significantly increased the mRNA expression of Bcl-2 by 87.2, 132.0, 139.6, 143.4, 164.2 and 165.3\%, respectively, compared with the cells exposed to propofol without dexmedetomidine pretreatment $(\mathrm{P}<0.05$; Fig. 3A and B). Similarly, increases of 35.1, 38.8, 58.0, 90.4, 108.1 and $135.1 \%$, respectively, were detected in the mRNA levels of BDNF in the dexmedetomidine-pretreated cells, compared to the propofol-treated cells $(\mathrm{P}<0.05$; Fig. $3 \mathrm{C}$ and $\mathrm{D})$. Consistent with the results of the semiquantitative RT-PCR analysis, dexmedetomidine significantly increased the protein levels of BDNF and Bcl-2, compared with the cells exposed to propofol 
A

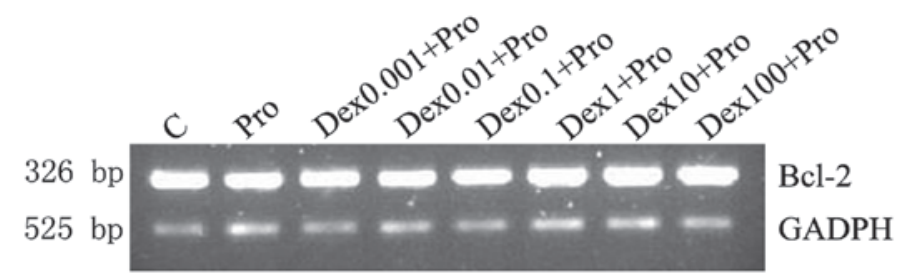

B

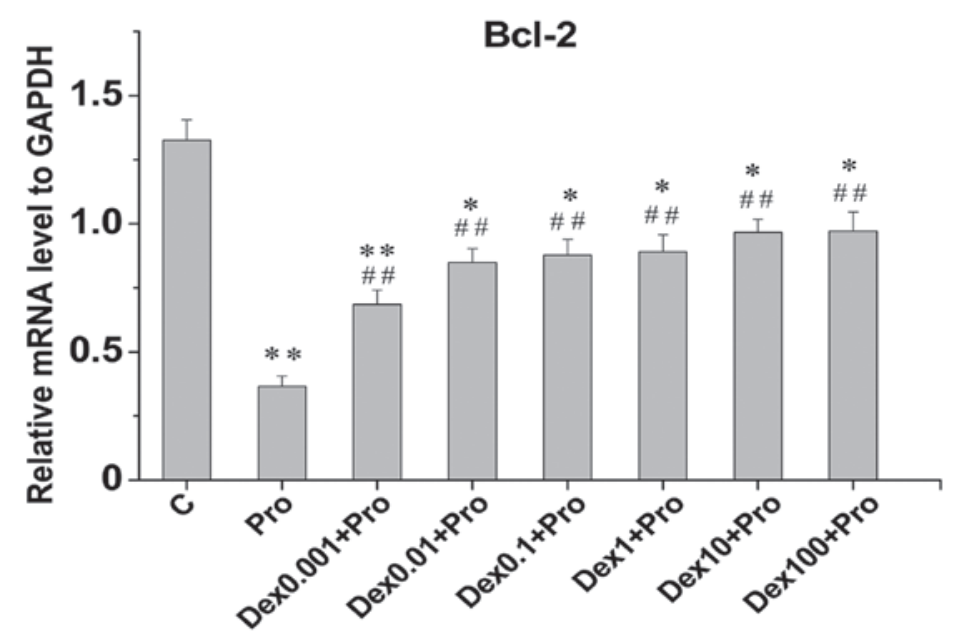

C

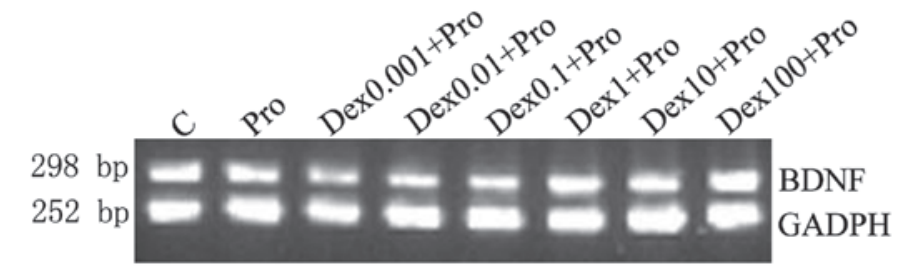

D

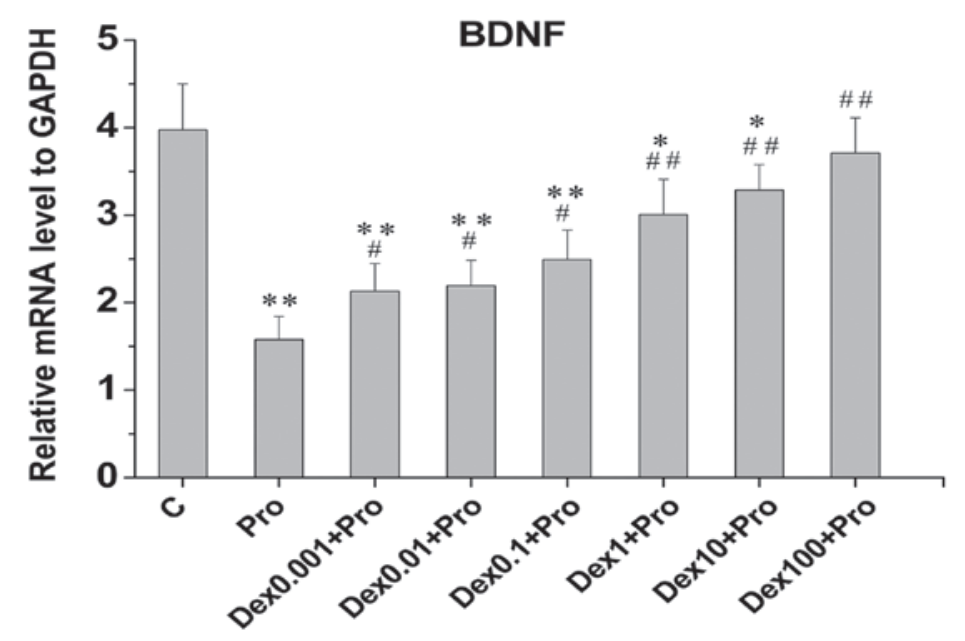

Figure 3. Dex attenuates Pro-induced reductions in the mRNA expression levels of BDNF and Bcl-2 in immature hippocampal neurons. The cells (8 days in vitro $)$ were exposed to Pro $(100 \mu \mathrm{M}, 3 \mathrm{~h})$ in the absence or presence of Dex pretreatment $(0.001,0.01,0.1,1,10$ and $100 \mu \mathrm{M})$. The mRNA levels of (A and B) BDNF and (C and D) Bcl-2, were determined using semiquantitative reverse transcription-quantitative polymerase chain reaction analysis, in which the densities of bands for BDNF and Bcl-2 were determined and standardized to the mRNA levels of GAPDH. Data are presented as the mean \pm standard deviation of at least three independent experiments. ${ }^{*} \mathrm{P}<0.05$ and ${ }^{* *} \mathrm{P}<0.01$, compared with group $\mathrm{C}$; ${ }^{*} \mathrm{P}<0.05$ and ${ }^{\# \#} \mathrm{P}<0.01$, compared with group Pro. Dex, dexmedetomidine; C, control; Pro, propofol; BDNF; brain-derived neurotrophic factor; Bcl-2, B-cell lymphoma 2.

without dexmedetomidine pretreatment $(\mathrm{P}<0.05$; Fig. 4C and $\mathrm{D}$ ). The protein levels of p-CREB in the dexmedetomidine-pretreated cells were also significantly upregulated by $18.4,54.7,60.4,80.7,116.3$ and $121.2 \%$, respectively, compared with those in the propofol-treated cells in the absence of dexmedetomidine pretreatment $(\mathrm{P}<0.05$; Fig. 4B).

\section{Discussion}

The present study used neuronal cultures from the rat hippocampus to investigate the neuroprotective effect of dexmedetomindine against propofol-induced neurotoxicity in vitro. The results showed that $100 \mu \mathrm{M}$ propofol triggered 
A

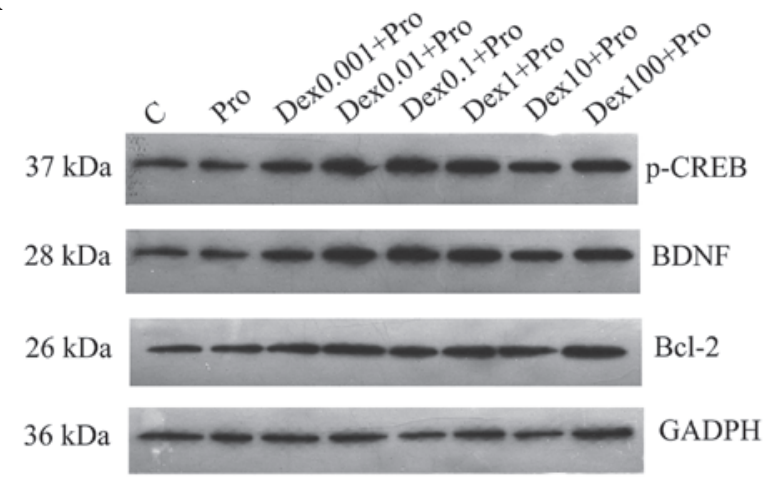

C

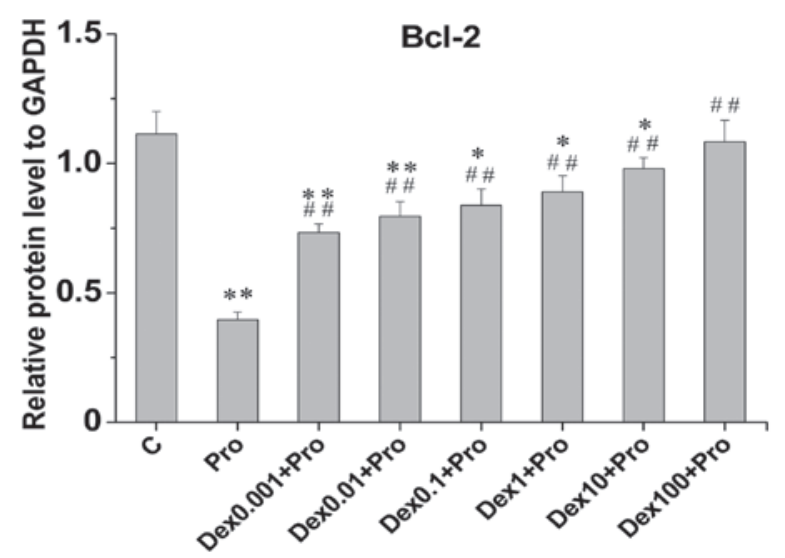

B

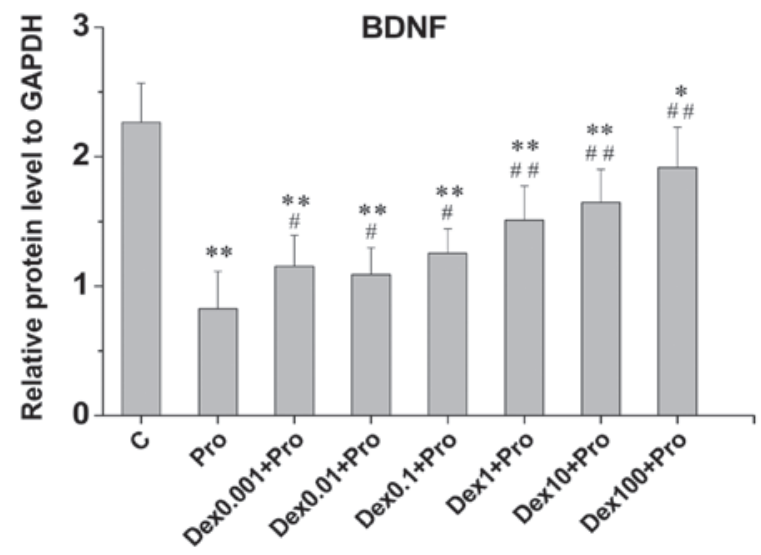

D

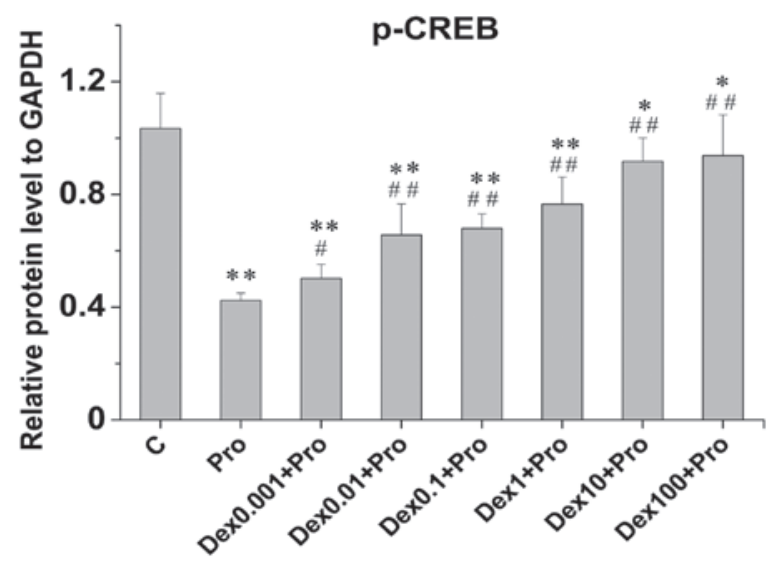

Figure 4. Dex attenuates Pro-induced reductions in the protein expression levels of p-CREB, BDNF and Bcl-2 in immature hippocampal neurons. The cell ( 8 days in vitro) were exposed to Pro $(100 \mu \mathrm{M}, 3 \mathrm{~h})$ in the absence or presence of Dex pretreatment $(0.001,0.01,0.1,1,10$ and $100 \mu \mathrm{M})$. Protein levels of Bcl-2, BDNF and p-CREB were examined using western blot analysis. (A) Western blotting bands of all groups of hippocampal neurons. Protein levels of (B) p-CREB, (C) BDNF and (D) Bcl-2 were standardized by the expression of GAPDH. Data are presented as the mean \pm standard deviation and compared using one-way analysis of variance. ${ }^{*} \mathrm{P}<0.05$ and ${ }^{* *} \mathrm{P}<0.01$, compared with group $\mathrm{C}$; ${ }^{\#} \mathrm{P}<0.05$ and ${ }^{\# \#} \mathrm{P}<0.01$, compared with group Pro. Dex, dexmedetomidine; C, control; Pro, propofol; BDNF; brain-derived neurotrophic factor; Bcl-2, B-cell lymphoma 2; phosphorylated-cyclic-AMP response element binding protein.

immature hippocampal neuron (DIV 8) apoptosis, reduced neuronal viability and caused a reduction in the levels of p-CREB, BDNF and Bcl-2. Pretreatment of the DIV 8 neurons with $0.001-100 \mu \mathrm{M}$ dexmedetomindine prior to propofol exposure attenuated the propofol-induced neuronal apoptosis and reduction in neuronal viability, and was accompanied by increases in the levels of p-CREB, Bcl-2 and BDNF. These in vitro data indicated that dexmedetomidine prevented propofol-induced neurotoxicity in neuronal cultures from the rat hippocampus, which was associated with upregulation in the expression levels of $\mathrm{p}-\mathrm{CREB}, \mathrm{BDNF}$ and $\mathrm{Bcl}-2$.

Several studies have demonstrated that the administration of anesthetics, including isoflurane, ketamine and propofol, trigger neuronal apoptosis in developing rodent and NHP brains $(6,16-18)$. In the present study DIV 8 hippocampal neurons were used to represent a model of immature and developing neurons. Primary neurons at DIV7-8 are sensitive to anesthetics, and it has been shown that high doses of propofol induce marked neuroapoptosis in the neonatal brain $(4,19)$. In aggregated cell cultures, propofol at a high concentration of $10 \mu \mathrm{g} / \mathrm{ml}$ has been reported to produce neurotoxic effects on neurons (20). In the present study, the concentration of propofol selected $(100 \mu \mathrm{M})$ was sufficiently high enough to induce neurotoxicity, which was confirmed by preliminary experiments. Therefore, the hippocampal neurons at DIV 8 in the present study were treated with $100 \mu \mathrm{M}$ propofol in the follow-up experiment. The present study found that exposure of immature neurons to $100 \mu \mathrm{M}$ propofol for $3 \mathrm{~h}$ resulted in a significant reduction in neuronal viability and increase in the percentage of apoptotic cells, as indicated using the CCK-8 assay and flow cytometric analysis. This indicated that propofol exposure induced neuronal injury and apoptosis. These results are in accordance with previous in vitro reports on the neurotoxicity of propofol $(3,21)$. In the present study, propofol exposure provoked neuronal damage in vitro and demonstrated that this damage was attenuated by dexmedetomidine.

Dexmedetomidine has been shown to exert neuroprotective effects against neurotoxicity induced by anesthetics, including isoflurane and ketamine, in the developing brain $(9,10,22,23)$. However, there is no data available on whether dexmedetomidine provides neuroprotective properties against propofol-induced neurotoxicity in the developing brain. The present study assessed the neuroprotective effect 
of dexmedetomidine on neuronal cultures from the rat hippocampus. Based on the previous studies of Sanders et al (22) and Dahmani et al (24), the concentration range of dexmedetomidine was extended $(0.001-100 \mu \mathrm{M})$ in the present study. A marked increase in neuronal viability and reduction in the rate of neuronal apoptosis were observed in the cells pretreated with dexmedetomidine at concentrations between 0.001 and $100 \mu \mathrm{M}$. This suggested that dexmedetomidine attenuated the negative effects of propofol on neuronal viability and survival in the rat hippocampal neuronal cultures. Unlike a previous study by Laudenbach et al (25), which found that dexmedetomidine concentrations of 10 and $100 \mu \mathrm{M}$ provided less neuroprotection, compared with lower concentrations in vitro, maximal neuroprotection was observed at the highest concentration of dexmedetomidine $(100 \mu \mathrm{M})$ in the present study, as revealed using an CCK-8 assay and flow cytometric analysis. At this concentration, no significant differences in neuronal viability or apoptosis were found between the dexmedetomidine + propofol group and the control group, which indicated that $100 \mu \mathrm{M}$ dexmedetomidine caused complete reversal of the neuronal injury induced by propofol in vitro. Other in vivo studies have reported that dexmedetomidine at a high concentration attenuates the neurotoxicity caused by ketamine completely (23), but it is unable to completely attenuate the cortical injury caused by isoflurane (22). This discrepancy may be due to differences in the experimental approach and anesthetics used in these studies. The present study also investigated whether alterations in the levels of p-CREB, Bcl-2 and BDNF are involved in the neuroprotective effects of dexmedetomidine.

The mechanisms underlying the neuroprotective effects of dexmedetomidine remain to be fully elucidated. Previous studies have indicated that dexmedetomidine-induced neuroprotection is mediated partially by the activation of $\alpha 2$-adrenergic receptors (9), the c-Jun N-terminal kinase and p38 mitogen-activated protein kinase pathways (26), the phosphoinositide 3-kinase/Akt pathway (10), and the extracellular signal-regulated kinase (ERK)1/2 pathways (27). CREB, a transcription factor family member, can be activated by activation of ERK1/2 and Akt pathways (28). The activation of CREB was has been suggested to regulate the expression of the genes associated with neuronal survival, synaptic plasticity and memory maintenance $(29,30)$. Whether CREB pathways are involved in the neuroprotective effects of dexmedetomidine remain to be fully elucidated. CREB is located upstream of neurotrophins. The phosphorylation of CREB at Ser-133 (p-CREB) regulates the transcription of pro-survival factors, including BDNF and Bcl-2. Increases in the levels of BDNF and $\mathrm{p}$-CREB are observed in the process of neuroprotection following neuronal injury $(31,32)$. Bcl-2, as a key apoptosis inhibitory protein, determines the mitochondrial response to apoptotic stimuli, and protects cells from apoptosis (33). In the present study, semiquantitative RT-PCR and western blot analyses revealed decreases in the expression levels of p-CREB, BDNF and Bcl-2 following propofol administration. Dexmedetomidine treatment prior to propofol exposure significantly attenuated the propofol-induced neuroapoptosis and increased the expression levels of $\mathrm{p}-\mathrm{CREB}, \mathrm{BDNF}$ and Bcl-2 compared with the propofol only group. These findings suggested that propofol-induced neurotoxicity was associated with lower levels of p-CREB, BDNF and Bcl-2, and revealed a link between the upregulation of p-CREB, BDNF and $\mathrm{Bcl}-2$ and the protective effect of dexmedetomindine against propofol-induced neuronal damage caused in developing neurons.

The present study had a number of limitations. Firstly, whether dexmedetomidine itself is directly toxic to immature neurons and whether it inhibits developmental neuroapoptosis, a normal physiologic process in the developing brain, were not investigated. However, several studies have shown that dexmedetomidine itself does not induce neuronal apoptosis in the developing brain of rodents $(9,22)$ or NHPs $(34)$. Secondly, due to the size of the subjects, in vivo experiments were not performed immediately following the in vitro experiments. Therefore, careful in vivo experiments are required to confirm the in vitro findings obtained in the present study to determine the correlation between the concentrations and protective efficacy of dexmedetomidine, and to investigate the underlying molecular mechanisms in the developing brain.

In conclusion, the present study demonstrated that dexmedetomidine attenuated propofol-induced neurotoxicity in neuronal cultures from the rat hippocampus, and this was associated with an increase in the levels of p-CREB, BDNF and $\mathrm{Bcl}-2$. The results of the present study contribute to the increasing body of evidence that dexmedetomidine exerts neuroprotective properties.

\section{Acknowledgements}

This study was supported by the National Natural Science Foundation of China (grant nos. 81373498 and 81060277), the Science Study and Technology Development Program of Guangxi (grant no. 1355005-4-2) and the Science and Technology Research Project of Guangxi University (grant no. 2013ZD014).

\section{References}

1. Sall JW, Stratmann G, Leong J, Woodward E and Bickler PE: Propofol at clinically relevant concentrations increases neuronal differentiation but is not toxic to hippocampal neural precursor cells in vitro. Anesthesiology 117: 1080-1090, 2012.

2. Kahraman S, Zup SL, McCarthy MM and Fiskum G: GABAergic mechanism of propofol toxicity in immature neurons. J Neurosurg Anesthesiol 20: 233-240, 2008.

3. Spahr-Schopfer I, Vutskits L, Toni N, Buchs PA, Parisi L and Muller D: Differential neurotoxic effects of propofol on dissociated cortical cells and organotypic hippocampal cultures. Anesthesiology 92: 1408-1417, 2000.

4. Cattano D, Young C, Straiko MM and Olney JW: Subanesthetic doses of propofol induce neuroapoptosis in the infant mouse brain. Anesth Analg 106: 1712-1714, 2008.

5. Pesić V, Milanović D, Tanić N, Popić J, Kanazir S, Jevtović-Todorović V and Ruzdijić S: Potential mechanism of cell death in the developing rat brain induced by propofol anesthesia. Int J Dev Neurosci 27: 279-287, 2009.

6. Creeley C, Dikranian K, Dissen G, Martin L, Olney J and Brambrink A: Propofol-induced apoptosis of neurones and oligodendrocytes in fetal and neonatal rhesus macaque brain. $\mathrm{Br}$ J Anaesth 110 (Suppl 1): i29-i38, 2013.

7. Afonso J and Reis F: Dexmedetomidine: Current role in anesthesia and intensive care. Rev Bras Anestesiol 62: 118-133, 2012.

8. Paris A, Mantz J, Tonner PH, Hein L, Brede M and Gressens P: The effects of dexmedetomidine on perinatal excitotoxic brain injury are mediated by the alpha2A-adrenoceptor subtype. Anesth Analg 102: 456-461, 2006. 
9. Sanders RD, Xu J, Shu Y, Januszewski A, Halder S, Fidalgo A, Sun P, Hossain M, Ma D and Maze M: Dexmedetomidine attenuates isoflurane-induced neurocognitive impairment in neonatal rats. Anesthesiology 110: 1077-1085, 2009.

10. Li Y, Zeng M, Chen W, Liu C, Wang F, Han X, Zuo Z and Peng S: Dexmedetomidine reduces isoflurane-induced neuroapoptosis partly by preserving PI3K/Akt pathway in the hippocampus of neonatal rats. PloS One 9: e93639, 2014.

11. Lu LX, Yon JH, Carter LB and Jevtovic-Todorovic V: General anesthesia activates BDNF-dependent neuroapoptosis in the developing rat brain. Apoptosis 11: 1603-1615, 2006.

12. Hwang L, Choi IY, Kim SE, Ko IG, Shin MS, Kim CJ, Kim SH, Jin JJ, Chung JY and Yi JW: Dexmedetomidine ameliorates intracerebral hemorrhage-induced memory impairment by inhibiting apoptosis and enhancing brain-derived neurotrophic factor expression in the rat hippocampus. Int J Mol Med 31: 1047-1056, 2013.

13. Yan M, Dai H, Ding T, Dai A, Zhang F, Yu L, Chen G and Chen Z: Effects of dexmedetomidine on the release of glial cell line-derived neurotrophic factor from rat astrocyte cells. Neurochem Int 58: 549-557, 2011.

14. Bell MT, Puskas F, Bennett DT, Herson PS, Quillinan N, Fullerton DA and Reece TB: Dexmedetomidine, an $\alpha-2 \mathrm{a}$ adrenergic agonist, promotes ischemic tolerance in a murine model of spinal cord ischemia-reperfusion. $\mathrm{J}$ Thorac Cardiovasc Surg 147: 500-506, 2014

15. Lesuisse C, Qiu D, Böse CM, Nakaso K and Rupp F: Regulation of agrin expression in hippocampal neurons by cell contact and electrical activity. Brain Res Mol Brain Res 81: 92-100, 2000.

16. Bercker S, Bert B, Bittigau P, Felderhoff-Müser U, Bührer C, Ikonomidou C, Weise M, Kaisers UX and Kerner T: Neurodegeneration in newborn rats following propofol and sevoflurane anesthesia. Neurotox Res 16: 140-147, 2009.

17. Brambrink AM, Evers AS, Avidan MS, Farber NB, Smith DJ, Zhang X, Dissen GA, Creeley CE and Olney JW: Isoflurane-induced neuroapoptosis in the neonatal rhesus macaque brain. Anesthesiology 112: 834-841, 2010.

18. Young C, Jevtovic-Todorovic V, Qin YQ, Tenkova T, Wang H, Labruyere J and Olney JW: Potential of ketamine and midazolam, individually or in combination, to induce apoptotic neurodegeneration in the infant mouse brain. Br J Pharmacol 146: 189-197, 2005

19. Fredriksson A, Pontén E, Gordh T and Eriksson P: Neonata exposure to a combination of $\mathrm{N}$-methyl-D-aspartate and gamma-aminobutyric acid type A receptor anesthetic agents potentiates apoptotic neurodegeneration and persistent behavioral deficits. Anesthesiology 107: 427-436, 2007.

20. Honegger P and Matthieu JM: Selective toxicity of the general anesthetic propofol for GABAergic neurons in rat brain cel cultures. J Neurosci Res 45: 631-636, 1996.

21. Vutskits L, Gascon E, Tassonyi E and Kiss JZ: Clinically relevant concentrations of propofol but not midazolam alter in vitro dendritic development of isolated gamma-aminobutyric acid-positive interneurons. Anesthesiology 102: 970-976, 2005 .
22. Sanders RD, Sun P, Patel S, Li M, Maze M and Ma D: Dexmedetomidine provides cortical neuroprotection: Impact on anaesthetic-induced neuroapoptosis in the rat developing brain. Acta Anaesthesiol Scand 54: 710-716, 2010

23. Duan X, Li Y, Zhou C, Huang L and Dong Z: Dexmedetomidine provides neuroprotection: Impact on ketamine-induced neuroapoptosis in the developing rat brain. Acta Anaesthesiol Scand 58: 1121-1126, 2014.

24. Dahmani S, Rouelle D, Gressens P and Mantz J: Characterization of the postconditioning effect of dexmedetomidine in mouse organotypic hippocampal slice cultures exposed to oxygen and glucose deprivation. Anesthesiology 112: 373-383, 2010.

25. Laudenbach V, Mantz J, Lagercrantz H, Desmonts JM, Evrard P and Gressens P: Effects of alpha(2)-adrenoceptor agonists on perinatal excitotoxic brain injury: Comparison of clonidine and dexmedetomidine. Anesthesiology 96: 134-141, 2002.

26. Liao Z, Cao D, Han X, Liu C, Peng J, Zuo Z, Wang F and Li Y: Both JNK and P38 MAPK pathways participate in the protection by dexmedetomidine against isoflurane-induced neuroapoptosis in the hippocampus of neonatal rats. Brain Res Bull 107: 69-78, 2014.

27. Zhu YM, Wang CC, Chen L, Qian LB, Ma LL, Yu J, Zhu MH, Wen CY, Yu LN and Yan M: Both PI3K/Akt and ERK1/2 pathways participate in the protection by dexmedetomidine against transient focal cerebral ischemia/reperfusion injury in rats. Brain Res 1494: 1-8, 2013.

28. Lonze BE and Ginty DD: Function and regulation of CREB family transcription factors in the nervous system. Neuron 35 : 605-623, 2002

29. Impey S, McCorkle SR, Cha-Molstad H, Dwyer JM, Yochum GS, Boss JM, McWeeney S, Dunn JJ, Mandel G and Goodman RH: Defining the CREB regulon: A genome-wide analysis of transcription factor regulatory regions. Cell 119: 1041-1054, 2004

30. Kandel ER: The molecular biology of memory storage: A dialogue between genes and synapses. Science 294: 1030-1038, 2001.

31. Zhang L, Zhao H, Zhang X, Chen L, Zhao X, Bai X and Zhang J: Nobiletin protects against cerebral ischemia via activating the p-Akt, p-CREB, BDNF and Bcl-2 pathway and ameliorating BBB permeability in rat. Brain Res Bull 96: 45-53, 2013.

32. Huang W, Cao J, Liu X, Meng F, Li M, Chen B and Zhang J: AMPK plays a dual role in regulation of $\mathrm{CREB} / \mathrm{BDNF}$ pathway in mouse primary hippocampal cells. J Mol Neurosci 56: 782-788, 2015

33. Cory S, Huang DC and Adams JM: The Bcl-2 family: Roles in cell survival and oncogenesis. Oncogene 22: 8590-8607, 2003.

34. Koo E, Oshodi T, Meschter C, Ebrahimnejad A and Dong G: Neurotoxic effects of dexmedetomidine in fetal cynomolgus monkey brains. J Toxicol Sci 39: 251-262, 2014. 